\title{
Correlação Entre Padrão Postural em Jovens Praticantes do Atletismo
}

\section{Correlation of the Postural Standard in Young Track and Field Practitioners}

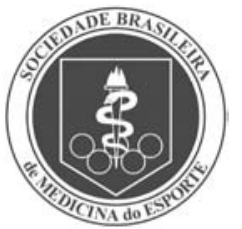

Artigo Original

\author{
Fábio do Nascimento Bastos² \\ Carlos Marcelo Pastre ${ }^{1,2}$ \\ Jayme Netto Júnior \\ Luiz Carlos Marques Vanderlei ${ }^{1,2}$ \\ Guaracy Carvalho Filho ${ }^{3}$ \\ Rosangela Akemi Hoshi² \\ Carlos Roberto Padovani 2,4
}

\section{Departamento de Fisioterapia. Faculdade de Ciências e Tecnologia/ Unesp. Presidente Prudente, SP - Brasil. \\ 2. Programa de Mestrado em Fisioterapia. Faculdade de Ciências e Tecnologia/Unesp. Presidente Prudente, SP - Brasil. \\ 3. Departamento de Ortopedia e Traumatologia. Famerp, São José do Rio Preto, SP - Brasil. \\ 4. Departamento de Bioestatística. Instituto de Biociências/Unesp. Botucatu, SP - Brasil.}

\section{Endereço para correspondência} Fábio do Nascimento Bastos Rua Padre João Goetz, 1.111, apto. 13, bloco A. Jardim Esplanada - 19061-460 Presidente Prudente, SP - Brasil. E-mail: bastosfisio@yahoo.com.br

Submetido em: 08/11/2008

Versão final recebida em: 16/04/2009 Aceito em: 14/05/2009

\begin{abstract}
RESUMO
O estudo objetivou observar o alinhamento corporal de jovens atletas praticantes de atletismo e analisar a associação de tais padrões dentro e entre os grupos de provas desta modalidade. Participaram 63 atletas de ambos os sexos e o protocolo de coleta para análise postural baseou-se na observação e registro fotográfico com marcações nos principais acidentes ósseos visando à verificação do alinhamento dessas estruturas. Os segmentos considerados para análise foram: tronco, pelve, joelho e tornozelo. Foi utilizado para análise dos dados o teste de Goodman para contrastes entre e dentro de proporções binomiais. Os resultados mostraram que saltadores (58,33\%) e arremessadores/lançadores (50,00\%) apresentam o tronco em posição mais equilibrada que atletas de resistência $(5,56 \%)$. Na região do quadril, arremessadores/lançadores apresentaram predomínio de pelve com rotação esquerda (66,67\%), enquanto nos saltadores foi observada elevada frequência de anteversão pélvica (58,33\%). A observação do joelho revelou altas taxas de normalidade e recurvatum em fundistas, valgo nos arremessadores/lançadores $(50,00 \%)$ e varo para os demais grupos. Para a articulação do tornozelo, nota-se que, exceto os saltadores, os grupos apresentaram predominância de valgismo. Conclui-se que, para atender às características particulares de cada prova, níveis distintos de postura são estabelecidos.
\end{abstract}

Palavras-chave: postura, adolescente, esportes.

\begin{abstract}
The aim of this study was to observe the body alignment of young athletes practicing athletics and analyze the combination of such patterns within and between groups of this modality. The sample was composed of 63 athletes of both sexes and the postural analysis protocol was based on observation and photographic record with markings on bone's accidents to verify the alignment of these structures. The segments considered in the analysis were: trunk, pelvis, knee and ankle. The Goodmann's test for contrasts between and within binomial proportions was used for data analysis. The results showed that jumpers (58.33\%) and throwers/ launchers (50.00\%) presented the trunk in more balanced position than resistance athletes (5.56\%). At the hip region, throwers/ launchers presented predominance of pelvis with left rotation (66.67\%), while in jumpers was observed high frequencies for pelvic anteversion (58,33\%). The knee observation revealed that resistance athletes presented normality and recurvatum, valgus in throwers/ launchers $(50,00 \%)$ and varus for the other groups. For the ankle, except for jumpers, all groups showed predominance of valgism. It is concluded that to reach the specific characteristics of each population, different levels of posture are established.
\end{abstract}

Keywords: posture, adolescent, sports.

\section{INTRODUÇÃO}

A prática de atividade motora a partir de métodos sistematizados de treinamento gera adaptações orgânicas necessárias para otimização do desempenho. Elas podem tornar-se definitivas, dependendo do tempo de exposição, tipo de técnica, modalidade e especificidade dos gestos atléticos ${ }^{(1,2)}$. Contudo, não raramente, ocorrem desequilíbrios musculares com consequentes alterações posturais, onde o organismo se reorganiza por meio de cadeias de compensação procurando uma resposta adaptativa a essa desarmonia ${ }^{(3,4)}$.

Nesse sentido, o esporte praticado desde a infância e adolescência pode levar o atleta a adaptações referentes aos gestos treinados, resultando em modificações no alinhamento corporal. A partir disso, pesquisadores têm utilizado tal fato como indicador de características próprias para determinadas modalidades ou como indicadores de predisposição às lesões desportivas ${ }^{(5,6)}$.
Os métodos de análise postural têm crescido ao longo dos últimos 15 anos, sendo aplicada particularmente no âmbito da medicina desportiva, na qual proporciona melhor compreensão da fisiologia da postura, bem como dos efeitos biomecânicos responsáveis para aquisição da mesma $a^{(5)}$.

A avaliação da postura estática é uma das maneiras válidas e aceitas para definir o perfil e características do aparelho locomotor. Consiste na análise do alinhamento corporal por um observador; os dados anotados revelam a simetria do corpo e seus segmentos ${ }^{(3,7)}$.

Contudo, embora comuns no âmbito clínico, são escassas as publicações que analisam a postura como característica física particular para determinada prática esportiva. Dessa forma, a partir da falta de informações sobre o referido tema e da facilidade de obtenção de dados junto à elite do atletismo brasileiro, pelos autores desta pesquisa, entendeu-se como pertinente empreender investigação sobre o assunto. 
Assim, constituiu-se como objetivo do presente estudo a observação do alinhamento corporal de jovens atletas praticantes de atletismo e a análise da associação de tais padrões dentro e entre os grupos de provas pertencentes ao respectivo esporte.

\section{MÉTODOS}

\section{Casuística do estudo}

Foram investigados 63 atletas de ambos os sexos, sendo para o sexo masculino - idade: 16,11 \pm 0,87 anos; peso: 72,41 \pm 7,04kg; estatura: 1,74 \pm 0,03m; e tempo de treinamento: 4,11 $\pm 0,84$ anos. Para os sujeitos do sexo feminino - idade: 16,21 \pm 0,79 anos; peso: 61,89 \pm 8,41 kg; estatura: 1,63 $\pm 0,08 \mathrm{~m}$; e tempo de treinamento: 2,58 \pm 0,77 anos. Todos participaram do Campeonato Brasileiro Caixa de Atletismo para Menores, 2007, ocorrido na cidade de São Paulo/SP.

Os atletas e seus respectivos responsáveis assinaram um termo de consentimento livre e esclarecido onde fica assegurada a privacidade dos mesmos. A pesquisa foi aprovada pelo Comitê de Ética em Pesquisa da Faculdade de Medicina de São José do Rio Preto - Famerp. Processo no 3.935/2002.

\section{Definição das variáveis de estudo}

O protocolo de coleta de dados para realização do exame físico e observacional foi baseado no estudo de Netto Jr. et al. ${ }^{(7)}$, buscando observações do alinhamento global dos segmentos do corpo, e Bienfait(3), empregando palpação na identificação de acidentes ósseos para visualizar e constatar alterações, sendo utilizado, para tanto, simetrógrafo da marca Carci $^{\circledR}$, fio de prumo, câmera fotográfica digital da marca Sony ${ }^{\circledR}$ DSC-P92 Cyber Shot de 5.0 megapixels e demarcadores cutâneos.

As observações após as marcações dos acidentes ósseos foram realizadas à distância que variava entre $2 \mathrm{~m}$ e $2,5 \mathrm{~m}$, dependendo da estatura do atleta, a mesma em que se fixava a câmera fotográfica para posterior registro de imagem.

\section{Coleta e análise dos dados}

Adotou-se como meta para coleta de dados a competição nacional mais importante para os participantes. $O$ atleta foi colocado em posição ortostática no simetrógrafo com calcanhares afastados $(5 \mathrm{~cm})$ e pés abduzidos cerca de 15 graus, buscando-se as assimetrias nos planos frontal, sagital e transversal ${ }^{(7)}$. Os participantes apresentaram-se apenas com "roupa para banho de piscina", enquanto o avaliador realizou as análises da postura in vivo - simultaneamente, um auxiliar efetuou o registro fotográfico das posições correspondentes à interpretação do pesquisador.

Os segmentos considerados para análise foram: tronco e pelve nos planos sagital, frontal e transversal, joelho no plano sagital e frontal, e o tornozelo no plano frontal, na qual se buscou a observação da qualidade de alinhamento dos segmentos. Os achados foram anotados em formulário próprio para facilitar a tabulação dos dados para posterior análise estatística.

De acordo com Tunes e Cote Gil(8), os modelos específicos para análise de segmentos ou pares de segmentos corporais tendem a fornecer informações detalhadas. No entanto, apenas essa iniciativa não implica necessariamente precisão, pois a subjetividade do observador pode comprometer a qualidade do registro efetuado.

Para minimizar possível viés de interpretação do avaliador, a documentação fotográfica foi utilizada posteriormente para confirmação, por outro especialista, dos diagnósticos emitidos pelo avaliador; nesse caso, o segundo avaliador não tomou conhecimento prévio das conclusões emitidas pelo primeiro. Desse modo, as informações sobre as alterações da postura consideradas para o estudo são aquelas que resultaram do consenso de ambos os avaliadores.

\section{Análise estatística}

Foi utilizado para a análise dos dados o teste de Goodman ${ }^{(9)}$ para contrastes entre e dentro de proporções binomiais. Assim, nas tabelas, para representação da significância dos achados foram utilizadas letras, como segue: i) as minúsculas indicam a comparação de grupos fixada à categoria de resposta; ii) as maiúsculas indicam a comparação de categorias de resposta dentro do grupo. Todas as conclusões foram discutidas para 5\% de significância estatística.

\section{RESULTADOS}

No plano sagital, saltadores (58,33\%) e arremessadores/lançadores (50,00\%) apresentam tronco em posição mais equilibrada que atletas de resistência (5,56\%). Na relação intragrupos, para os atletas de resistência e saltadores houve predomínio do tronco em desequilíbrio anterior. O grupo arremessos/lançamentos apresentou maior frequência para o tronco em posição de equilíbrio e com desvio anterior. No plano frontal, foi verificado que o grupo resistência apresentou diferença estatística no que se refere ao desequilíbrio do tronco para o lado direito (38,88\%) e para o esquerdo (55,56\%). No plano transverso, também não foi notada qualquer diferença entre os grupos avaliados, porém, dentro do grupo arremessos/lançamentos houve maior frequência para postura do tronco equilibrado $(61,11 \%)$ (tabela 1).

Tabela 1. Distribuição de frequências, absoluta e relativa (\%), dos participantes segundo o grupo de provas e postura do tronco.

\begin{tabular}{|c|c|c|c|c|}
\hline \multirow{2}{*}{ Grupo } & \multicolumn{3}{|c|}{ Plano sagital } & \multirow[b]{2}{*}{ Total } \\
\hline & Equilibrado & Anterior & Posterior & \\
\hline Velocidade & $7(46,67 \%)^{\mathrm{abA}}$ & $6(40,00 \%)^{\mathrm{aA}}$ & $2(13,33 \%)^{\mathrm{aA}}$ & 15 \\
\hline Resistência & $1(5,56 \%)^{\mathrm{AA}}$ & $12(66,66 \%)^{\mathrm{aB}}$ & $5(27,78 \%)^{\mathrm{AB}}$ & 18 \\
\hline Saltos & $7(58,33 \%)^{\mathrm{bB}}$ & $4(33,34 \%)^{a A B}$ & $1(8,33 \%)^{\mathrm{aA}}$ & 12 \\
\hline \multirow[t]{3}{*}{$\begin{array}{l}\text { Arremessos/ } \\
\text { Lançamentos }\end{array}$} & $9(50,00 \%)^{\mathrm{bB}}$ & $8(44,44 \%)^{\mathrm{aB}}$ & $1(5,56 \%)^{\mathrm{aA}}$ & 18 \\
\hline & \multicolumn{3}{|c|}{ Plano frontal } & \\
\hline & Equilibrado & Direito & Esquerdo & \\
\hline Velocidade & $3(20,00 \%)^{\mathrm{aA}}$ & $4(26,67 \%)^{a A}$ & $8(53,33 \%)^{\mathrm{aA}}$ & 15 \\
\hline Resistência & $1(5,56 \%)^{\mathrm{aA}}$ & $7(38,88 \%)^{\mathrm{aB}}$ & $10(55,56 \%)^{\mathrm{aB}}$ & 18 \\
\hline Saltos & $2(16,66 \%)^{\mathrm{aA}}$ & $5(41,67 \%)^{\mathrm{aA}}$ & $5(41,67 \%)^{\mathrm{aA}}$ & 12 \\
\hline \multirow[t]{3}{*}{$\begin{array}{l}\text { Arremessos/ } \\
\text { Lançamentos }\end{array}$} & $4(22,22 \%)^{a A}$ & $5(27,78 \%)^{\mathrm{aA}}$ & $9(50,00 \%)^{\mathrm{aA}}$ & 18 \\
\hline & \multicolumn{3}{|c|}{ Plano transversal } & \\
\hline & Equilibrado & Direito & Esquerdo & \\
\hline Velocidade & $6(40,00 \%)^{\mathrm{aA}}$ & $4(26,67 \%)^{a A}$ & $5(33,33 \%)^{\mathrm{aA}}$ & 15 \\
\hline Resistência & $10(55,56 \%)^{\mathrm{aA}}$ & $3(16,66 \%)^{a A}$ & $5(27,78 \%)^{\mathrm{AA}}$ & 18 \\
\hline Saltos & $3(25,00 \%)^{a A}$ & $5(41,67 \%)^{\mathrm{AA}}$ & $4(33,33 \%)^{a A}$ & 12 \\
\hline $\begin{array}{l}\text { Arremessos/ } \\
\text { Lançamentos }\end{array}$ & $11(61,11 \%)^{\mathrm{aB}}$ & $2(11,11 \%)^{\mathrm{AA}}$ & $5(27,78 \%)^{\mathrm{AB}}$ & 18 \\
\hline
\end{tabular}

Teste de Goodman para contrastes entre e dentro de populações multinomiais, onde as letras maiúsculas são para relacionar as linhas, sendo $A<B$ e as letras minúsculas, as colunas, sendo $a<b$. Os valores entre parênteses são as frequências relativas de cada valor.

Para a pelve (tabela 2), verifica-se que no plano sagital não foi observada diferença entre os grupos em relação à distribuição dos casos. Já dentro dos grupos, foi observado que na pelve as posições normal $(41,67 \%)$ e em anteversão (58,33\%) são estatisticamente predominantes para as provas de salto. 
No plano frontal, a análise intergrupos não revelou diferenças, enquanto que a comparação intragrupos mostrou desvios em todos os grupos. Rotação pélvica à esquerda, no plano transversal, foi estatisticamente maior dentro do grupo de arremessos.

Tabela 2. Distribuição de frequências, absoluta e relativa (\%), dos participantes segundo o grupo de provas e postura da pelve.

\begin{tabular}{|c|c|c|c|c|}
\hline \multirow{2}{*}{ Grupo } & \multicolumn{3}{|c|}{ Plano sagital } & \multirow{2}{*}{ Tota } \\
\hline & Normal & Anteversão & Retroversão & \\
\hline Velocidade & $6(40,00 \%)^{\mathrm{aA}}$ & $7(46,67 \%)^{\mathrm{aA}}$ & $2(13,33 \%)^{a A}$ & 15 \\
\hline Resistência & $4(22,22 \%)^{a A}$ & $8(44,45 \%)^{\mathrm{aA}}$ & $6(33,33 \%)^{\mathrm{aA}}$ & 18 \\
\hline Saltos & $5(41,67 \%)^{\mathrm{aB}}$ & $7(58,33 \%)^{a \mathrm{~B}}$ & $0(0,00 \%)^{\mathrm{aA}}$ & 12 \\
\hline \multirow[t]{3}{*}{$\begin{array}{l}\text { Arremessos/ } \\
\text { Lançamentos }\end{array}$} & $5(27,78 \%)^{\mathrm{aA}}$ & $7(38,89 \%)^{\mathrm{aA}}$ & $6(33,33 \%)^{\mathrm{aA}}$ & 18 \\
\hline & \multicolumn{3}{|c|}{ Plano frontal } & \\
\hline & Normal & Direita mais alta & Esquerda mais alta & \\
\hline Velocidade & $0(0,00 \%)^{\mathrm{aA}}$ & $7(46,67 \%)^{a \mathrm{~B}}$ & $8(53,33 \%)^{a \mathrm{~B}}$ & 15 \\
\hline Resistência & $0(0,00 \%)^{\mathrm{aA}}$ & $7(38,89 \%)^{a \mathrm{~B}}$ & $11(61,11 \%)^{\mathrm{aB}}$ & 18 \\
\hline Saltos & $1(8,33 \%)^{\mathrm{aA}}$ & $5(41,67 \%)^{\mathrm{aB}}$ & $6(50,00 \%)^{a \mathrm{~B}}$ & 12 \\
\hline \multirow[t]{3}{*}{$\begin{array}{l}\text { Arremessos/ } \\
\text { Lançamentos }\end{array}$} & $1(5,56 \%)^{\mathrm{aA}}$ & $8(44,44 \%)^{a \mathrm{~B}}$ & $9(50,00 \%)^{\mathrm{aB}}$ & 18 \\
\hline & \multicolumn{3}{|c|}{ Plano transversal } & \\
\hline & Normal & Rotação direita & Rotação esquerda & \\
\hline Velocidade & $6(40,00 \%)^{\mathrm{aA}}$ & $3(20,00 \%)^{a A}$ & $6(40,00 \%)^{a A}$ & 15 \\
\hline Resistência & $4(22,22 \%)^{a A}$ & $9(50,00 \%)^{a A}$ & $5(27,78 \%)^{a A}$ & 18 \\
\hline Saltos & $3(25,00 \%)^{a A}$ & $5(41,67 \%)^{a A}$ & $4(33,33 \%)^{a A}$ & 12 \\
\hline $\begin{array}{l}\text { Arremessos/ } \\
\text { Lançamentos }\end{array}$ & $2(11,11 \%)^{\mathrm{aA}}$ & $4(22,22 \%)^{a A}$ & $12(66,67 \%)^{a B}$ & 18 \\
\hline
\end{tabular}

Teste de Goodman para contrastes entre e dentro de populaçôes multinomiais, onde as letras maiúsculas săo para relacionar as linhas, sendo $\mathrm{A}<\mathrm{B}$ e as letras minúsculas, as colunas, sendo $\mathrm{a}<\mathrm{b}$. Os valores entre parênteses são as frequências relativas de cada valor.

A análise do joelho (tabela 3) mostra que no plano sagital, em todos os grupos, foi observado predomínio da normalidade. Ainda nesse plano, atletas de resistência apresentaram joelhos em recurvatum. A análise no plano frontal revelou presença de varo em velocistas, fundistas e saltadores, enquanto que no grupo de arremessos/lançamentos, o predomínio foi de normalidade e valgismo.

Tabela 3. Distribuição de frequências, absoluta e relativa (\%), dos participantes segundo o grupo de provas e postura do joelho.

\begin{tabular}{|c|c|c|c|c|}
\hline \multirow{2}{*}{ Grupo } & \multicolumn{3}{|c|}{ Plano sagital } & \multirow{2}{*}{ Total } \\
\hline & Normal & Flexo & Recurvatum & \\
\hline Velocidade & $11(73,33 \%)^{\mathrm{aC}}$ & $4(26,67 \%)^{\mathrm{aB}}$ & $0(0,00 \%)^{\mathrm{aA}}$ & 15 \\
\hline Resistência & $11(61,11 \%)^{\mathrm{aB}}$ & $1(5,56 \%)^{\mathrm{aA}}$ & $6(33,33 \%)^{a B}$ & 18 \\
\hline Saltos & $8(66,67 \%)^{a B}$ & $1(8,33 \%)^{\mathrm{aA}}$ & $3(25,00 \%)^{\mathrm{aAB}}$ & 12 \\
\hline \multirow[t]{3}{*}{$\begin{array}{l}\text { Arremessos/ } \\
\text { Lançamentos }\end{array}$} & $12(66,66 \%)^{\mathrm{aB}}$ & $3(16,67 \%)^{\mathrm{aA}}$ & $3(16,67 \%)^{\mathrm{aA}}$ & 18 \\
\hline & \multicolumn{3}{|c|}{ Plano frontal } & \\
\hline & Normal & Valgo & Varo & \\
\hline Velocidade & $4(26,67 \%)^{\mathrm{aAB}}$ & $2(13,33 \%)^{\mathrm{abA}}$ & $9(60,00 \%)^{\mathrm{bB}}$ & 15 \\
\hline Resistência & $3(16,67 \%)^{\mathrm{aA}}$ & $0(0,00 \%)^{\mathrm{aA}}$ & $15(83,33 \%)^{\mathrm{bB}}$ & 18 \\
\hline Saltos & $1(8,33 \%)^{\mathrm{aA}}$ & $2(16,67 \%)^{\mathrm{abA}}$ & $9(75,00 \%)^{\mathrm{bB}}$ & 12 \\
\hline $\begin{array}{l}\text { Arremessos/ } \\
\text { Lançamentos }\end{array}$ & $8(44,44 \%)^{\mathrm{aB}}$ & $9(50,00 \%)^{\mathrm{bB}}$ & $1(5,56 \%)^{\mathrm{aA}}$ & 18 \\
\hline
\end{tabular}

Teste de Goodman para contrastes entre e dentro de populações multinomiais, onde as letras maiúsculas são para relacionar as linhas, sendo $\mathrm{A}<\mathrm{B}$ e as letras minúsculas, as colunas, sendo $\mathrm{a}<\mathrm{b}$. Os valores entre parênteses são as frequências relativas de cada valor.
Para articulação do tornozelo, não foram observadas diferenças significantes entre os grupos, sendo os resultados apresentados na tabela 4. Nota-se que, exceto saltadores, todos grupos apresentaram predominância de tornozelo valgo.

Tabela 4. Distribuição de frequências, absoluta e relativa (\%), dos participantes segundo o grupo de provas e postura do tornozelo.

\begin{tabular}{l|c|c|c|c}
\hline \multirow{2}{*}{ Grupo } & \multicolumn{3}{|c|}{ Plano frontal } & \multirow{2}{*}{ Total } \\
\cline { 2 - 4 } & Normal & Valgo & Varo & \\
\hline Velocidade & $3(20,00 \%)^{\mathrm{aB}}$ & $12(80,00 \%)^{\mathrm{aC}}$ & $0(0,00 \%)^{\mathrm{aA}}$ & 15 \\
\hline Resistência & $3(16,67 \%)^{\mathrm{aA}}$ & $12(66,66 \%)^{\mathrm{aB}}$ & $3(16,67 \%)^{\mathrm{AA}}$ & 18 \\
\hline Saltos & $4(33,33 \%)^{\mathrm{aA}}$ & $6(50,00 \%)^{\mathrm{aA}}$ & $2(16,67 \%)^{\mathrm{AA}}$ & 12 \\
\hline $\begin{array}{l}\text { Arremessos/ } \\
\text { Lançamentos }\end{array}$ & $4(22,22 \%)^{\mathrm{aA}}$ & $13(72,22 \%)^{\mathrm{aB}}$ & $1(5,56 \%)^{\mathrm{aA}}$ & 18 \\
\hline
\end{tabular}

Teste de Goodman para contrastes entre e dentro de populações multinomiais, onde as letras maiúsculas são para relacionar as linhas, sendo $A<B$ e as letras minúsculas, as colunas, sendo $a<b$. Os valores entre parênteses são as frequências relativas de cada valor.

\section{DISCUSSÃO}

O treinamento esportivo gera adaptações morfológicas e funcionais do sistema musculoesquelético em consequência da especificidade de cada prova. Tal fenômeno pode caracterizar grupos de atletas submetidos a estímulos motores de mesma natureza sob a ótica antropométrica, incluindo a postura corporal. Assim, entende-se como pertinente investigar padrões de postura nos esportes, sobretudo na adolescência.

Embora o tema da presente comunicação seja de conhecimento geral no âmbito das ciências do esporte, ainda são escassos os estudos referentes a alterações posturais, o que, associado à falta de padronização nos registros de informações, impossibilita uma discussão mais aprofundada sobre o assunto. Dessa forma, as possibilidades de comparação de resultados com outras pesquisas, assim como o levantamento de hipóteses para explicar os achados, tornam-se restritas, determinando uma fragilidade a ser considerada.

Além disso, o método utilizado para avaliação postural neste estudo, conforme sugerido por Netto Jr. et al. ${ }^{(7)}$, pode ser destacado como outra limitação. Atualmente, têm-se utilizado programas de computador, como o Software para Avaliação Postural (SAPO), para auxiliar nas avaliações, proporcionando medidas quantitativas de comprimentos, ângulos e desvios, entre outras variáveis dos segmentos corporais de um sujeito(10).

Especificamente sobre os achados, destaca-se o seguinte: atletas saltadores e arremessadores/lançadores apresentaram maiores frequências para postura equilibrada do tronco em relação ao grupo de resistência, no qual foi observada maior tendência para tronco anteriorizado (66,66\%). A necessidade da estabilização desse segmento para realização do gesto atlético pode ser a causa dessa condição(11-13).

Foi observado desequilíbrio anterior do tronco em atletas de resistência. Segundo Bricot ${ }^{(14)}$, desequilíbrios dessa natureza podem ser consequência de hiperlordose lombar, causada por valgismo de tornozelo, padrões posturais encontrados no presente estudo. Ressalta-se que curvaturas da coluna vertebral não foram analisadas, porém, foi observada alta taxa para pelve em anteversão que, por sua vez, provoca aumento da curvatura lombar devido a compensações biomecânicas ${ }^{(3)}$.

O grupo arremessos/lançamentos apresentou maiores frequências para pelve com rotação esquerda. Teixeira(15) descreve que o movimento de flexão do quadril associado ao movimento oposto do braço parece ter função estabilizadora precedente ao movimento de rotação pélvica durante o lançamento. Isso ocorre em virtude do forte deslocamento anterior do centro de gravidade durante a realização de uma ação mo- 
tora de arremesso em trajetória balística, caracterizando uma estratégia de controle para manter o centro de gravidade sobre a base de apoio.

Em todos os grupos houve predominância de desvio pélvico no plano transversal, divergindo dos resultados de Netto Jr. et al. ${ }^{(7)}$ que, em estudo com atletas velocistas, verificaram alterações nos planos frontal e horizontal, com rotação da pelve à direita e lado oposto mais elevado. Esses autores ${ }^{(7)}$ ainda explicam que a corrida em curva faz o atleta solicitar de maneira excessiva o quadril para manutenção da velocidade com simultânea mudança de direção em função de forças resultantes.

Observou-se que saltadores apresentam altas taxas para anteversão pélvica, que também pode estar associada à especificidade(11). Para o salto, o atleta exige que seu centro de gravidade se desloque anteriormente, promovendo alcance de máxima velocidade horizontal. Assim, hiperextensão da coluna lombar, associada à extensão de membros inferiores, pode determinar esse desequilíbrio ${ }^{(12)}$.

Além disso, foi observada a predominância de valgismo de tornozelo em todos os grupos, o que pode ser explicado de maneira mais aceitável pela hipótese de Netto Jr. et al. ${ }^{(7)}$. Destaca-se a existência de bloqueio do mecanismo de propriocepção neuromuscular do segmento analisado, com consequente redução da sustentação dos ossos do pé, que desabam medialmente sob a ação da descarga do peso corporal.

Para os velocistas e saltadores foram observadas altas taxas de joeIho varo, divergindo dos achados de Pastre et al. ${ }^{(2)}$, que verificaram essa condição, porém, em atletas de resistência. Esses autores mencionam a relação desses padrões com o elevado volume de trabalho e fadiga proporcionado pelo treinamento. Essa condição pode ocasionar estresse articular e redução na absorção de impactos, convergindo para as observações de Pastre et al..11), os quais mencionam essa articulação como a mais acometida por lesões.

Algumas lesões desportivas podem estar relacionadas com os desequilíbrios posturais que os atletas adquirem desde o início da prática atlética ${ }^{(15)}$. Ressalta-se que, no atletismo, grande parte dos praticantes inicia seus treinamentos antes dos 15 anos de idade, faixa etária em que ainda não ocorreu a maturação óssea. Nesse sentido, a instalação da postura em varo do joelho em 71,43\% dos atletas deste estudo pode ser resultado da constante sobrecarga exigida nos treinamentos, podendo comprimir as epífises de crescimento na porção medial, consequentemente, causando desenvolvimento assimétrico das mesmas ${ }^{(16,17)}$.

Modalidades que requerem corrida proporcionam tensão nas unidades miotendíneas, devido ao comprimento dos músculos isquiotibiais e a sua atividade contrátil. Esses aspectos, juntamente com seu formato biarticular e o desequilíbrio de forças em relação ao quadríceps femoral, podem ser causas de importantes retrações musculares, o que poderia explicar alterações na postura, como joelho em recurvatum ${ }^{(11,14,18)}$. Tais observações foram encontradas, na presente investigação, no grupo resistência, ao passo que entre os velocistas não foram verificados os mesmos resultados.

Outra hipótese a ser considerada, em relação ao recurvatum do joelho, é que pode ser causado por retração da musculatura estática da perna. O músculo sóleo tensionado, devido a sua origem na linha solear da tíbia, leva esse segmento no sentido anteroposterior, aumentando o ângulo tibiotársico e, como consequência, a hiperextensão do joelho ${ }^{(3)}$.

Por fim, destaca-se que os achados do presente estudo somam-se à escassa literatura referente às alterações posturais em adolescentes praticantes de atletismo de alta performance. Uma vez que tais alterações podem gerar desequilíbrios no aparelho musculoesquelético ${ }^{(3,6)}$, torna-se importante a avaliação precoce, como medida profilática, no sentido de zelar pela integridade física do atleta, bem como melhorar sua performance. Essas medidas podem, ainda, contribuir para prevenir instalação de processos crônicos, causado pelos treinamentos de alta intensidade de esforço, tornando-se um aspecto importante em se tratando de aumento de rendimento ${ }^{(19,20)}$.

\section{CONCLUSÃO}

A partir das observações e comparações realizadas neste estudo, conclui-se que, para característica particular nas diversas modalidades do atletismo, são adotados níveis distintos de postura. O grupo velocidade e o de resistência apresentam varo de joelho e tornozelo valgo.

Os especialistas em saltos apresentaram predominantemente tronco equilibrado, anteversão pélvica e joelho varo. Por fim, os arremessadores/lançadores apresentaram tronco em posição de equilíbrio, rotação esquerda de pelve, joelho normal e tornozelo valgo.

Contudo, espera-se que estudos sobre avaliação postural sejam realizados, principalmente no âmbito desportivo, utilizando protocolos mais completos associados a testes de retrações musculares e nível de força, de modo a garantir resultados mais fidedignos, permitindo comparar com outros estudos de mesma natureza para que, posteriormente, possa ser traçado o perfil postural das diversas populações atléticas.

Todos os autores declararam não haver qualquer potencial conflito de interesses referente a este artigo.

\section{REFERÊNCIAS BIBLIOGRÁFICAS}

1. Yoshitomi SK, Tanaka C, Duarte M, Lima F, Morya E, Hazime F. Respostas posturais à perturbação externa inesperada em judocas de diferentes níveis de habilidade. Rev Bras Med Esporte. 2006;12:159-63.

2. Pastre CM, Carvalho Filho G, Monteiro HL, Netto Júnior J, Padovani CR. Lesões desportivas no atletismo: comparação entre informaçōes obtidas em prontuários e inquéritos de morbidade referida. Rev Bras Med Esporte. 2004;10:1-8.

3. Bienfait M. Os desequilíbrios estáticos: fisiologia, patologia e tratamento fisioterápico. São Paulo: Summus, 1995.

4. Paillard T, Noé F, Rivière T, Marion V, Montoya R, Dupui P. Postural performance and strategy in the unipedal stance of soccer players at different levels of competition. J Athl Train. 2006;41:172-6.

5. Viton JM, Mesure S, Bensoussan L, Mattei JP, Coudreuse JM, Delarque A. Analyse de la posture et du mouvement et médecine du sport [Posture and movement analysis and sports medicine]. Ann Readapt Med Phys. 2004;47:258-62

6. Ribeiro CZP, Akashi PMH, Sacco ICN, Pedrinelli A. Relationship between postural changes and injuries of the locomotor system in indoor soccer athletes. Rev Bras Med Esporte. 2003;9:98-103.

7. Netto Júnior J, Pastre CM, Monteiro HL. Alterações posturais em atletas brasileiros do sexo masculino que participaram de provas de potência muscular em competições internacionais. Rev Bras Med Esporte. 2004;10:195-8.

8. Tunes $\mathrm{E}$, Cote Gil HJ. Modelos de registro para a postura corporal em situações funcionais: uma revisão. Rev Bras Med Ocupacional. 1990;18:45-9.

9. Goodman LA. On simultaneous confidence intervals for multinomial proportions. Technometrics. $1965 ; 7: 247-54$
10. Ferreira EAG. Postura e controle postural: desenvolvimento e aplicação de método quantitativo de avaliação postural [Dissertação]. São Paulo: USP, Faculdade de Medicina, 2005.

11. Pastre CM, Carvalho Filho G, Monteiro HL, Netto Jr J, Padovani CR. Lesões desportivas na elite do atletismo brasileiro: estudo a partir de morbidade referida. Rev Bras Med Esporte. 2005:11:43-7.

12. Reis MC. Saltos. Barcelona: Editorial Sintes, 1970.

13. Weineck J. Anatomia aplicada ao esporte. São Paulo: Manole, 1990.

14. Bricot B. Posturologia. Ed. Ícone, 1999.

15. Teixeira LA. Coordenação intersegmentar em arremessos com diferentes demandas de precisão. Rev Paul Educ Fís. 1997;11:5-14.

16. Bowen RE, Dorey FJ, Moseley CF. Relative tibial and femoral varus as a predictor of progression of varus deformities of the lower limbs in young children. J Pediatr Orthop. 2002;22:105-11.

17. Iwamoto J, Abe H, Tsukimura Y, Wakano K. Relationship between radiographic abnormalities of lumbar spine and incidence of low back pain in high school rugby players: a prospective study. Scand J Med Sci Sports. 2005;15:163-8.

18. Laurino CFS, Lopes AD, Mano KS, Cohen M, Abdalla RJ. Lesões músculo-esqueléticas no atletismo. Rev Bras Ortop. 2000;35:364-8.

19. Detsch C, Luz AMH, Candotti CT, Oliveira DS, Lazaron F, Guimarães LK. Prevalência de alterações posturais em escolares do ensino médio em uma cidade no Sul do Brasil. Pan Am J Public Health. 2007;21:231-8.

20. Kratenova J, Zejglicova K, Maly M, Filipova V. Prevalence and risk factors of poor posture in school children in the Czech Republic. J Sch Health. 2007;77:131-7. 